\title{
Paediatric Rheumatology: Helen Foster and Paul A. Brogan (eds)
}

\section{Published by Oxford University Press, UK; Second Edition: 2018; ISBN: 978-0-19-873875-6}

\author{
Raju Khubchandani ${ }^{1,2}$
}

Received: 18 September 2018 / Accepted: 18 September 2018 / Published online: 16 October 2018

(C) Dr. K C Chaudhuri Foundation 2018

This book from the series of Oxford Specialist Handbooks in Pediatrics has something in it for everyone from the resident starting his career in pediatric rheumatology to the practicing pediatrician who is looking for a bird's eye view on a specific topic to even the senior practitioner looking to quickly access drug information, clinical guidelines and protocols in pediatric rheumatology.

Compiled and edited by two internationally renowned names in the pediatric rheumatology world, this multi author book draws mainly on contributors working in the UK and is fully endorsed by the British Society for Paediatric and Adolescent Rheumatology (BSPAR). Now in its second edition, Foster and Brogan have invited chapters from some of their international colleagues to give additional inputs in chapters on infections such as tuberculosis and HIV and their interface with rheumatology and immunization practices. This represents one of the important advances over the first edition.

While chapters 1 and 2 are aimed at initiating the reader to this branch which many medical students and practitioners are in awe of, the chapters on Juvenile idiopathic arthritis (JIA), systemic diseases and bone disorders clearly take the reader to the deep end providing systematic, practical, day-to-day, cur- rent approaches to the common rheumatology clinic issues. Notably all through, the book maintains a practical "Monday morning"approach to the problems without delving into the basic science that can often be daunting for a practitioner. Algorithms, text boxes and tables contribute to the ease of imbibing information.

I particularly found the chapters on specialised therapeutic approaches and clinical guidelines and protocols, highly helpful to check on drug doses, interactions and protocols on the go.

In maintaining their status as 'hand books' it is indeed light and palm sized and can easily fit into the apron pocket. However to cram so much of information in 553 pages, the publisher has to reduce the print size to a font which is just discernible, for example the table on page 194. The entire text is presented in bullet point format which some readers like while others prefer continuous text. The black and white reproduction of X-rays and clinical images (some of which have been repeated in color) leaves the only lacuna in this book which I otherwise have no hesitation in recommending as an outpatient clinic or ward companion.

I would be curious to know whether there is a Kindle edition?

Raju Khubchandani

rajukhubchandani@yahoo.co.in

1 Department of Pediatric Rheumatology, SRCC Childrens Hospital, Mumbai, India

2 Department of Pediatrics, Jaslok Hospital and Research Center, Breach Candy Hospital, Mumbai, India 